\title{
Folate-conjugated thermo-responsive micelles for tumor targeting
}

\author{
Jian Zhang, ${ }^{1,2}$ Dawei Deng, ${ }^{1}$ Hongyan Zhu, ${ }^{1}$ Youngro Byun, ${ }^{3}$ Victor C. Yang, ${ }^{2,3,4}$ Yueqing Gu ${ }^{1}$ \\ ${ }^{1}$ Department of Biomedical Engineering, School of Life Science and Technology, China Pharmaceutical University, Nanjing \\ 210009, People's Republic of China \\ ${ }^{2}$ Department of Pharmaceutical Sciences, College of Pharmacy, University of Michigan, Ann Arbor, Michigan 48109-1065 \\ ${ }^{3}$ Department of Molecular Medicine and Biopharmaceutical Sciences, Seoul National University, Seoul 151-702, South Korea \\ ${ }^{4}$ Tianjin Key Laboratory on Technologies Enabling Development of Clinical Therapeutics and Diagnostics, Tianjin Medical \\ University, Tianjin 300070, China
}

Received 7 February 2012; revised 1 April 2012; accepted 25 April 2012

Published online 24 July 2012 in Wiley Online Library (wileyonlinelibrary.com). DOI: 10.1002/jbm.a.34230

\begin{abstract}
Folate-conjugated and thermo-responsive poly $((\mathrm{N}$ isopropylacrylamide)-co- acrylamide-co-(octadecyl acrylate)co-(folate-(polyethylene glycol)-(acrylic acid))) (P(NIPA-coAAm-co-ODA-co-FPA)) micelles with mean diameter of about $60 \mathrm{~nm}$ and lower critical solution temperature (LCST) of about $39^{\circ} \mathrm{C}$ were synthesized by free radical random copolymerization. Single-factor tests of acrylamide and octadecyl acrylate were carried out to modulate micelles' LCST and diameter, respectively. LCST, diameter, and morphology of micelles were determined by UV-vis spectrophotometer, laser particle size analyzer, and transmittance electron microscope (TEM), respectively. Fluorescein was then used as a model drug to investigate the drug loading content of micelles. Micelles with maximum amount of octadecyl acrylate $(180 \mathrm{mg})$ were found
\end{abstract}

to yield drug loading content of $10.48 \%$. Near infrared dye No.10 was chosen as the tracer to monitor micelles in vivo. The targeting behaviors of micelles in folate receptor positive Bel-7402 tumor bearing nude mice were assessed by a selfconstructed near infrared imaging system. Results showed satisfactory targeting capability of the thermo-responsive micelles toward Bel-7402 tumors, and targeting accumulation could last for more than $96 \mathrm{~h}$, enabling P(NIPA-co-AAm-coODA-co-FPA) micelles to function as a diagnostic reagent as well as a targeted tumor therapy. (๑) 2012 Wiley Periodicals, Inc. J Biomed Mater Res Part A: 100A: 3134-3142, 2012.

Key Words: folate receptor, thermo-responsive, $\mathrm{N}$-isopropylacrylamide, micelle, Bel-7402 tumor

How to cite this article: Zhang J, Deng D, Zhu H, Byun Y, Yang VC, Gu Y. 2012. Folate-conjugated thermo-responsive micelles for tumor targeting. J Biomed Mater Res Part A 2012:100A:3134-3142.

\section{INTRODUCTION}

Due to the specific properties, micelles self-assembled from amphiphilic copolymers have attracted growing attention in biomedical applications. ${ }^{1}$ Such micelle-based drug delivery systems (DDS) possess many advantages over other particulate-based carriers ${ }^{2}$ including: (1) the long circulation of the particles in vivo; (2) the enhancement of solubility for incorporated hydrophobic drugs; (3) enhanced permeability and retention effect (EPR effect) in tumor tissues, the so-called passive targeting. ${ }^{3}$

Nevertheless, EPR-based passive targeting is not sufficient for tumor diagnosis or therapy. Consequently, micelles with active targeting capability could meet with this need and are thus highly desirable. ${ }^{4}$ Among these strategies, one approach that appears to be promising is to attach folic acid (folate) to the cargoes in achieving targeting selectivity to folate receptor positive tumors. ${ }^{5,6}$ As for polymeric carriers, $N$-isopropylacry- lamide (NIPA)-based polymers have recently drawn more attention owing to their intelligently responsive properties and low cytotoxicity. ${ }^{7-9}$ Indeed, they have been widely employed in drug and gene delivery. ${ }^{10-13}$ However, few studies paid attention to combining the targeting capability with the intelligently responsive property of NIPA-based polymers.

Aside from tumor therapy, early diagnosis of the tumor is also of great importance to reduce the mortality of patients. Near infrared imaging technique offers several advantages in early tumor diagnosis, such as noninvasiveness and real-time measurement. ${ }^{14}$ Furthermore, this technique is ideal for in vivo application because of the inherently low autofluorescence in the near infrared range (700-900 nm) and large Stokes shift between the excitation and emission bands. ${ }^{15}$ To this regard, near infrared dye No.10 (NIRD-10), a hydrophobic dye $(2-3 \mu \mathrm{g} / \mathrm{mL}$ in double distilled water) with maximum absorption at $769 \mathrm{~nm}$ and

Additional Supporting Information may be found in the online version of this article.

Correspondence to: V. C. Yang; e-mail: vcyang@umich.edu or Y. Gu; e-mail: cpuyueqing@163.com

Contract grant sponsor: Natural Science Foundation Committee of China; contract grant numbers: NSFC30371362, NSFC30672015, NSFC30700779

Contract grant sponsor: World Class University (WCU) program at the Department of Molecular Medicine and Biopharmaceutical Sciences, Seoul National University, South Korea; contract grant number: R31-2008-000-10103-01 


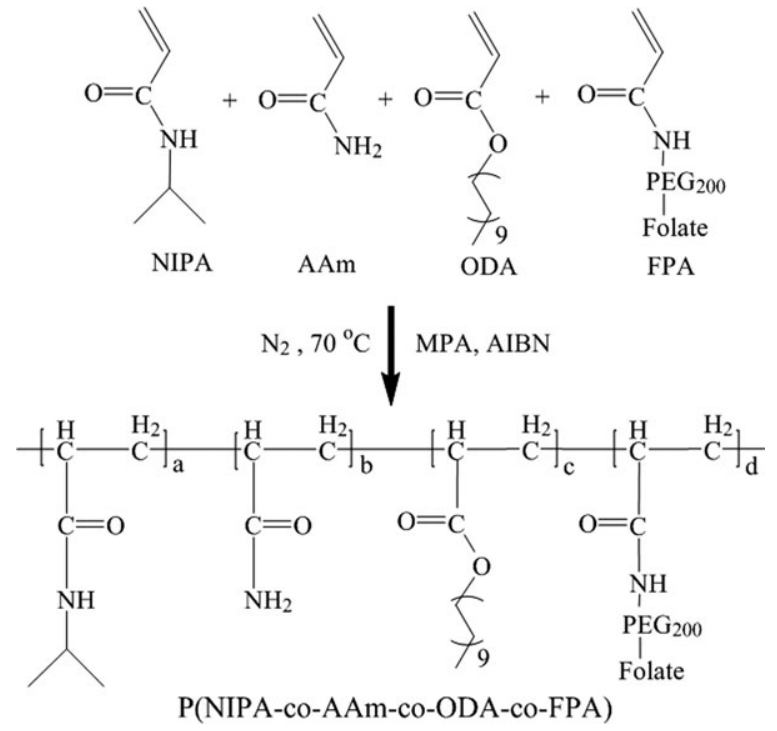

FIGURE 1. Schematic of the synthesis of P(NIPA-co-AAm-co-ODA-coFPA).

emission peak at $793 \mathrm{~nm}$ (Supporting Information Figs. 1 and 2), was selected as the tracer to monitor in vivo targeting of NIRD-10 loaded micelles.

In this report, folate-conjugated, NIPA-based poly $((\mathrm{N}$-isopropylacrylamide)-co-acrylamide- $\mathrm{co}$-(octadecylacrylate)-co-(folate-(polyethylene glycol)-(acrylic acid))) (P(NIPA-co- AAm-coODA-co-FPA)) micelles were synthesized. In vivo tumor targeting capability of the micelles was assessed in an athymic mouse model harboring folate receptor-expressing Bel-7402 tumors via near infrared imaging technique.

\section{MATERIALS AND METHODS Materials}

Amine-bis-(polyethylene glycol) 200 (Amino-PEG ${ }_{200}$ ) was supplied by Jenkem Technology (Peking, China). Folic acid (folate, MW 441), N,N'-dicyclohexylcarbodiimide (DCC) and $\mathrm{N}$-hydroxysuccinimide (NHS) were obtained from SigmaAldrich (Shanghai, China). $N$-isopropylacrylamide (NIPA, $\geq 99 \%$ in purity), acrylamide (AAm), octadecyl acrylate (ODA), acrylic acid (AA), 3-mercaptopropionic acid (MPA), and azobisisobutylnitrile (AIBN) were all purchased from Aldrich-Chemie (Steinheim, Germany) and used without further purification. Near infrared dye No.10 (NIRD-10) was obtained from Huangfan Technology Company (Beijing, China). Methyl thiazolyl tetrazolium (MTT) and other reagents for cell cultivation were from Gibco Life Technologies (Shanghai, China). Triethylamine, dimethyl formamide (DMF), dimethyl sulfoxideand (DMSO), and other organic solvents used in this study were acquired from Shanghai Chemical Company with analytical reagent grade (Shanghai, China). Water was double distilled.

\section{Synthesis of PEG-(acrylic acid) and folate-PEG-(acrylic acid)}

Amino- $\mathrm{PEG}_{200}$ was used as the crosslinker of folate and acrylic acid to the synthesis of folate-(polyethylene glycol)- (acrylic acid) (Folate-PEG-(Acrylic Acid), FPA). The synthesis of FPA consisted mainly of four steps (molar ratios for folate, amino- $\mathrm{PEG}_{200}$, and acrylic acid was maintained at 1:1.5:1.5): (1) activation of $\gamma$ carboxy group of folate by DCC/NHS chemistry to form folate-NHS; (2) conjugation of folate-NHS with amino- $\mathrm{PEG}_{200}$ to produce Folate-PEG-NH ; (3) activation of the carboxy group of acrylic acid by DCC/ NHS chemistry to form (acrylic acid)-NHS; (4) conjugation of folate-PEG- $\mathrm{NH}_{2}$ with (acrylic acid)-NHS to yield FPA. PEG(acrylic acid) was synthesized by conjugating (acrylic acid)NHS with amino-PEG 200 using the similar protocol described above for FPA.

\section{Synthesis of P(NIPA-co-AAm-co-ODA-co-FPA) (FNM)}

A series of Folate-conjugated, NIPA-based and Micelle-forming copolymers (FNM) with different feed compositions (Table I) were synthesized by free-radical random polymerization (Fig. 1). In detail, monomer NIPA (1500 mg), AAm (100-300 mg), ODA (0-180 mg), FPA (0 or $100 \mathrm{mg})$, and chain transfer agent 3-mercaptopropionic acid (15 $\mu \mathrm{L})$ were added to DMF (15 mL). The resulted mixture solution was then nitrogen-purged for $40 \mathrm{~min}$ at room temperature. Polymerization was then initiated by adding $30 \mathrm{mg}$ of azobisisobutylnitrile and incubated at $70^{\circ} \mathrm{C}$ for $4 \mathrm{~h}$ with nitrogen protection. FNM11 was synthesized by replacing FPA with the same amount of PEG-(Acrylic Acid) (Table I).

After polymerization, FNM copolymers were dialyzed (molecular weight cut off, $3.5 \mathrm{kDa}$ ) against double distilled water and self-assembled to form core-shell micelle structure via hydrophobic association (Fig. 2). After dialysis, the visible images of FNM04 (Table I) at $37^{\circ} \mathrm{C}$ and $45^{\circ} \mathrm{C}$ were taken to examine the thermo-responsive properties of the micelles. Absorption spectra of folate, FNM11, and FNM04 were also acquired by using a Lambda 35 UV-visible spectrophotometer (Perkin Elmer, America).

\section{Characterization of FNM}

The average diameters and distribution of FNM (FNM04, $07-10,5 \mathrm{mg} / \mathrm{mL}$ ) in double distilled water were measured by Mastersizer 2000 laser particle size analyzer (Malvern, British). Transmittance electron microscope (TEM) images were also taken by using a H-7000 TEM (Hitachi, Japan) with accelerated voltage of $75 \mathrm{kV}$.

To measure the lower critical solution temperature (LCST) of micelles, optical transmittances of FNM solutions

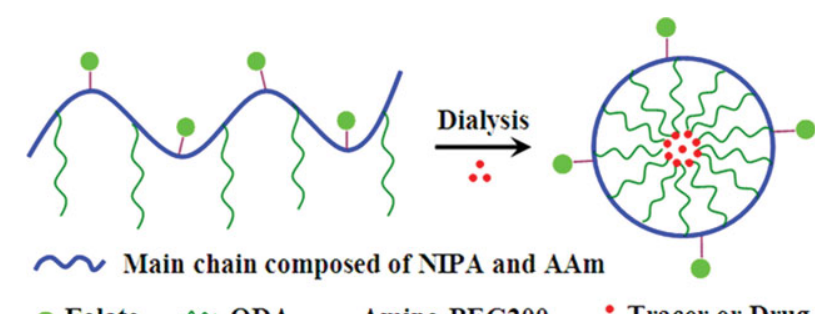

Folate $\quad \sim$ ODA - Amino-PEG200 $\therefore$ Tracer or Drug

FIGURE 2. Micelle formation of P(NIPA-co-AAm-co-ODA-co-FPA). [Color figure can be viewed in the online issue, which is available at wileyonlinelibrary.com.] 
TABLE I. Feed Compositions of the Synthesis of Micelles

\begin{tabular}{|c|c|c|c|c|c|c|c|}
\hline Samples & NIPA (mg) & $\mathrm{AAm}(\mathrm{mg})$ & ODA (mg) & FPA (mg) & $\operatorname{MPA}(\mu \mathrm{L})$ & AIBN (mg) & $\mathrm{DMF}(\mathrm{mL})$ \\
\hline FNM01 & 1500 & 100 & 120 & 100 & 20 & 30 & 15 \\
\hline FNM02 & 1500 & 150 & 120 & 100 & 20 & 30 & 15 \\
\hline FNM03 & 1500 & 200 & 120 & 100 & 20 & 30 & 15 \\
\hline FNM04 & 1500 & 250 & 120 & 100 & 20 & 30 & 15 \\
\hline FNM05 & 1500 & 300 & 120 & 100 & 20 & 30 & 15 \\
\hline FNM06 & 1500 & 250 & 0 & 100 & 20 & 30 & 15 \\
\hline FNM07 & 1500 & 250 & 60 & 100 & 20 & 30 & 15 \\
\hline FNM08 & 1500 & 250 & 90 & 100 & 20 & 30 & 15 \\
\hline FNM09 & 1500 & 250 & 150 & 100 & 20 & 30 & 15 \\
\hline FNM10 & 1500 & 250 & 180 & 100 & 20 & 30 & 15 \\
\hline FNM11 & 1500 & 250 & 120 & 0 & 20 & 30 & 15 \\
\hline
\end{tabular}

(FNM01-05, $5 \mathrm{mg} / \mathrm{mL}$ ) at various temperatures $(30$ to $50^{\circ} \mathrm{C}$ ) were measured at $500 \mathrm{~nm}$ by a UV-visible spectrophotometer $(n=3)$. At least $10 \mathrm{~min}$ of incubation was allowed for samples to reach equilibrium temperature.

To investigate the effect of ionic strength on the LCST of micelles, LCST of FNM04 was determined in different solutions $(5 \mathrm{mg} / \mathrm{mL}$ ) of various ionic strengths (Table II), including double distilled water, Hanks simulated body fluid (Table III), $0.9 \% \mathrm{NaCl}$ solution, and $1.8 \% \mathrm{NaCl}$ solution. Of the hydrophobic core, the amount of ODA residues could also affect LCST of FNM. LCST of FNM (FNM04, 07-10, $5 \mathrm{mg} / \mathrm{mL}$ ) in double distilled water was also measured using the same protocol.

\section{Drug loading of FNM}

Fluorescein was chosen as the model drug in this study due to its appropriate solubility in Hanks simulated body fluid. Fluorescein (10 mg) was loaded into FNM micelles via a previously established protocol. ${ }^{13}$ After incubation, centrifugation (14,000 r.p.m. $\times 20$ min, high speed centrifuge model 1612-1, Shanghai, China) was carried out to remove the unloaded fluorescein. The amount of fluorescein was quantified by measuring the absorbance of the solution at 484 $\mathrm{nm}$. The amount of entrapped model drug and drug loading content were then estimated according to the following definitions:

The amount of entrapped model drug $=$ (amount of added model drug) - (amount of unloaded model drug) - (amount of dissolved model drug in physiological saline); Drug loading content $(\%)=$ (amount of entrapped model drug) $/$ (amount of micelles and entrapped model drug) $\times 100 \%$.

Noncovalent labeling of FNM04 micelle with NIRD-10 The same protocol described in Section "drug loading of FNM" was employed to acquire the noncovalent labeling of FNM04 with NIRD-10, a hydrophobic dye with maximum

\section{TABLE II. Ionic Strengths of Solutions}

\begin{tabular}{lc}
\hline Solution & Ionic Strength \\
\hline Double distilled water & 0 \\
Hanks simulated body fluid & 0.1605 \\
$0.9 \% \mathrm{NaCl}$ solution (w/w) & 0.1513 \\
$1.8 \% \mathrm{NaCl}$ solution (w/w) & 0.3026
\end{tabular}

absorption at $769 \mathrm{~nm}$, and maximum emission at $793 \mathrm{~nm}$ (Supporting Information Figs. 1 and 2).

After labeling, absorbance and fluorescence spectra of free and entrapped NIRD-10 were measured by using a UVvisible spectrophotometer and S2000 spectrometer (Ocean Optics, USA), respectively. The photostability of free and entrapped NIRD-10 was estimated by measuring their fluorescence intensity under continuous exposure to the laser beam for $2 \mathrm{~h}$, with an interval of $5 \mathrm{~min}$ for data acquisition.

\section{MTT assay of NIRD-10 loaded FNM04}

Human Vein Endothelial Cell Line (ECV304) was chosen as the target cells for this MTT assay. Briefly, $200 \mu \mathrm{L}$ RPMI 1640 with ECV304 $\left(2.0 \times 10^{5}\right.$ cells $\left./ \mathrm{mL}\right)$ was added into each well of a 96 -well plate and incubated at $37^{\circ} \mathrm{C}$ for $24 \mathrm{~h}$ in humidified atmosphere containing $5 \% \mathrm{CO}_{2}$. The culture medium in each well was then replaced with $200 \mu \mathrm{L}$ RPMI 1640 medium containing NIRD-10 loaded FNM04 at predefined concentrations $(0 \mathrm{mg} / \mathrm{mL}, 0.02 \mathrm{mg} / \mathrm{mL}, 0.1 \mathrm{mg} / \mathrm{mL}$, $0.2 \mathrm{mg} / \mathrm{mL}, 0.5 \mathrm{mg} / \mathrm{mL}, 1.0 \mathrm{mg} / \mathrm{mL}$, and $2.0 \mathrm{mg} / \mathrm{mL}$ ). Medium without NIRD-10 loaded FNM04 was used as the control. The mixture was further incubated for $44 \mathrm{~h}$, and the medium of each well was replaced with $180 \mu \mathrm{L}$ fresh RPMI 1640 and $20 \mu \mathrm{L}$ MTT PBS solution ( $5 \mathrm{mg} / \mathrm{mL}, \mathrm{pH} 7.4$ ). After incubation for another $4 \mathrm{~h}$, the medium was further replaced with $150 \mu \mathrm{L}$ DMSO. The 96-well plate was then shaken at room temperature and the absorbance (A) of the solution in each well was measured at $570 \mathrm{~nm}$ and $630 \mathrm{~nm}$ with a Microplate Reader (Biorad, USA). The viable rate was calculated by using the following equation: Viable Rate = $A_{\text {treated }} / A_{\text {control }} \times 100 \%$, where $A_{\text {treated }}$ was obtained in the presence of NIRD-10 loaded FNM04, while $A_{\text {control }}$ was obtained in the absence of NIRD-10 loaded FNM04.

\section{In vivo targeting behaviors of FNM04 micelles}

Animal models. The athymic nude mice (nu/nu) used in this study, half male and half female of 4-6 weeks old and

TABLE III. Chemical Composition of Hanks Simulated Body Fluid ( $\mathrm{m} \mathrm{mol} / \mathrm{L}$ )

\begin{tabular}{rrrrrrrr}
\hline $\mathrm{Na}^{+}$ & $\mathrm{K}^{+}$ & $\mathrm{Ca}^{2+}$ & $\mathrm{Mg}^{2+}$ & $\mathrm{Cl}^{-}$ & $\mathrm{HCO}_{3}^{-}$ & $\mathrm{HPO}_{4}^{2-}$ & $\mathrm{SO}_{4}^{2-}$ \\
\hline 142.0 & 5.0 & 2.5 & 1.5 & 147.8 & 4.2 & 1.0 & 0.5 \\
\hline
\end{tabular}


weighing 18-22 g, were purchased from Charles River Laboratories (Shanghai, China). Each mouse was anesthetized in an isoflurane chamber before being injected subcutaneously in the right armpit with a PBS suspension containing $3 \times$ $10^{6}$ Bel-7402 tumor cells. Several days after inoculation, when the tumors reached $150-300 \mathrm{~mm}^{3}$ in volume, the mice were used for in vivo imaging studies.

All experiments were carried out in compliance with the Animal Management Rules of the Ministry of Health of the People's Republic of China (document no. 55, 2001) and the guidelines for the Care and Use of Laboratory Animals of China Pharmaceutical University.

\section{In vivo imaging test}

Fifteen athymic mice were evenly and randomly divided into three groups. Mice of group A, B, and C were injected via tail vein with NIRD-10, NIRD-10 loaded FNM11, and NIRD-10 loaded FNM04, respectively. Dose (5 $\mu \mathrm{g}$ ) for each group was calculated based on the amount of NIRD-10. The targeting behaviors of NIRD-10 loaded FNM04 were monitored using the previously introduced near infrared imaging system. ${ }^{8}$ For measurement, each mouse was imaged at predefined intervals ( $1 \mathrm{~min}, 2 \mathrm{~h}, 8 \mathrm{~h}, 24 \mathrm{~h}$, and $96 \mathrm{~h}$ ) postinjection.

Urine and feces of group C mice were also collected and imaged to trace the metabolism pathway of the FNM micelles. All of the mice were sacrificed at $96 \mathrm{~h}$ post-injection and their main organs (heart, liver, spleen, lung, kidney, muscle, and tumor) were collected for ex vivo imaging.

\section{RESULTS}

\section{Synthesis of FNM}

DCC/NHS chemistry was employed for the conjugation of folate, amino-PEG 200 , and acrylic acid via the formation of amide linkages. During FPA synthesis, all of the folate-containing compounds [folate, folate-NHS, folate-PEG- $\mathrm{NH}_{2}$, and folate-PEG-(Acrylic Acid)] were purified in acetone $(1: 4, \mathrm{v} /$ v) due to their poor solubility in acetone. The ninhydrin spray was employed to track of the synthesis process of FPA (Supporting Information Fig. 3), and results showed that FPA was precisely synthesized.

\section{Micelle formation and identification}

Visible images of FNM04 were taken at $37^{\circ} \mathrm{C} / 45^{\circ} \mathrm{C}$ (see Fig. 3 inset). The micelle solution was maize in color (caused by folate residues) and transparent at $37^{\circ} \mathrm{C}$ (below LCST), as letters behind the bottle could be visualized clearly. However, when the temperature was increased to $45^{\circ} \mathrm{C}$ (above LCST), the solution became opaque, as letters behind the bottle could no longer be seen. These results indicated that the micelles were thermo-responsive. The absorption spectrum of folate, FNM11, and FNM04 (Fig. 3) were also obtained. FNM11 micelle, without any specific chromophore, displayed no absorption peaks except nonspecific end absorption. On the other hand, FNM04 exhibited both the end absorption of FNM11 and unique absorption peaks of folate.

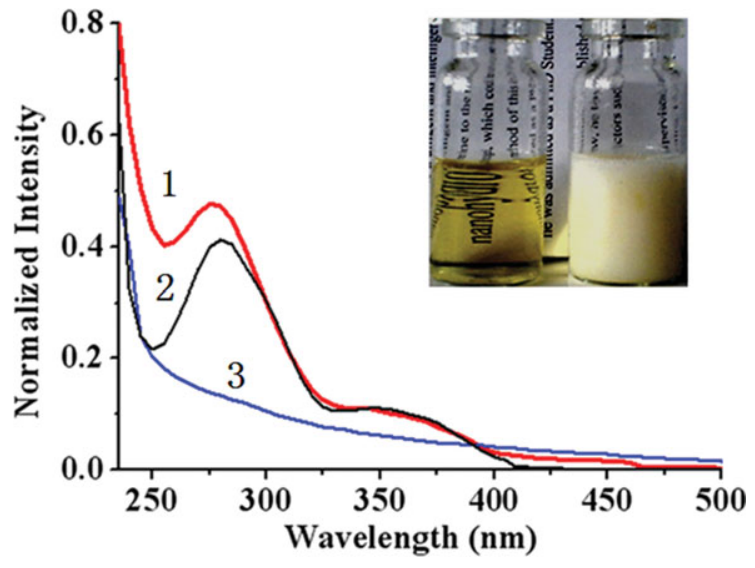

FIGURE 3. Absorption spectra of (1) P(NIPA-co-AAm-co-ODA-co-FPA); (2) folate and (3) P(NIPA-Co-AAm-co-ODA) (FNM11). Inset: visible images of $\mathrm{P}$ (NIPA-co-AAm-co-ODA-co-FPA) micelle at $37^{\circ} \mathrm{C}$ (below LCST, left bottle) and $45^{\circ} \mathrm{C}$ (above LCST, right bottle) in double distilled water. [Color figure can be viewed in the online issue, which is available at wileyonlinelibrary.com.]

\section{Diameter and morphology of FNM}

Figure 4(a) suggested that the diameters of FNM (FNM04, 0710 , with ODA amounts of $120 \mathrm{mg}, 60 \mathrm{mg}, 90 \mathrm{mg}, 150 \mathrm{mg}$, and $180 \mathrm{mg}$, respectively, Table I) altered as the amount of ODA (single-factor test of ODA). When ODA was $60 \mathrm{mg}$ (FNM07) micelles with an average diameter of about $200 \mathrm{~nm}$ [197.36 \pm 28.55 nm, Fig. 4(a)] was obtained. However, when the amount of ODA was increased to $180 \mathrm{mg}$ (FNM10), the diameter of the micelles decreased to only about $15 \mathrm{~nm}(14.04 \pm 2.19 \mathrm{~nm})$. As shown in Figure 4(b), the diameter distribution of FNM04 determined by laser particle size analyzer was quite narrow. In addition, the morphology of FNM04, measured by TEM [Fig. 4(c)], further confirmed the results of the particle size and distribution of FNM04. TEM picture also showed that micelles were well dispersed as individual particles with a spherical shape.

\section{LCST of FNM}

LCST of micelles (FNM01-05, with amount of AAm of 100, $150,200,250$, and $300 \mathrm{mg}$, respectively, Table I) were measured in this study. Figure 5(a) displayed the profiles of the transmittance of FNM solutions versus variation of the temperature. Results in Figure 5(b) indicated that LCST of micelles increased when increasing the amount of AAm. The sharp decrease of transmittance below/above LCST could validate that a satisfactory temperature sensitivity of the synthesized micelles was achieved.

Figure 5(c) depicted LCST of micelle dissolved in various solvents containing different ionic strength. FNM04 showed the highest LCST $\left(42^{\circ} \mathrm{C}\right)$ in double distilled water but a lower LCST $\left(39^{\circ} \mathrm{C}\right)$ in Hanks simulated body fluid and $0.9 \% \mathrm{NaCl}$ solution, both possessed a similar ionic strength. On the other hand, LCST of FNM04 in $1.8 \% \mathrm{NaCl}$ was only $37^{\circ} \mathrm{C}$, reducing by $5^{\circ} \mathrm{C}$ compared with that in double distilled water.

\section{Drug loading of FNM}

Fluorescein was chosen as the model drug to investigate the drug loading content of FNM04, due to its appropriate 

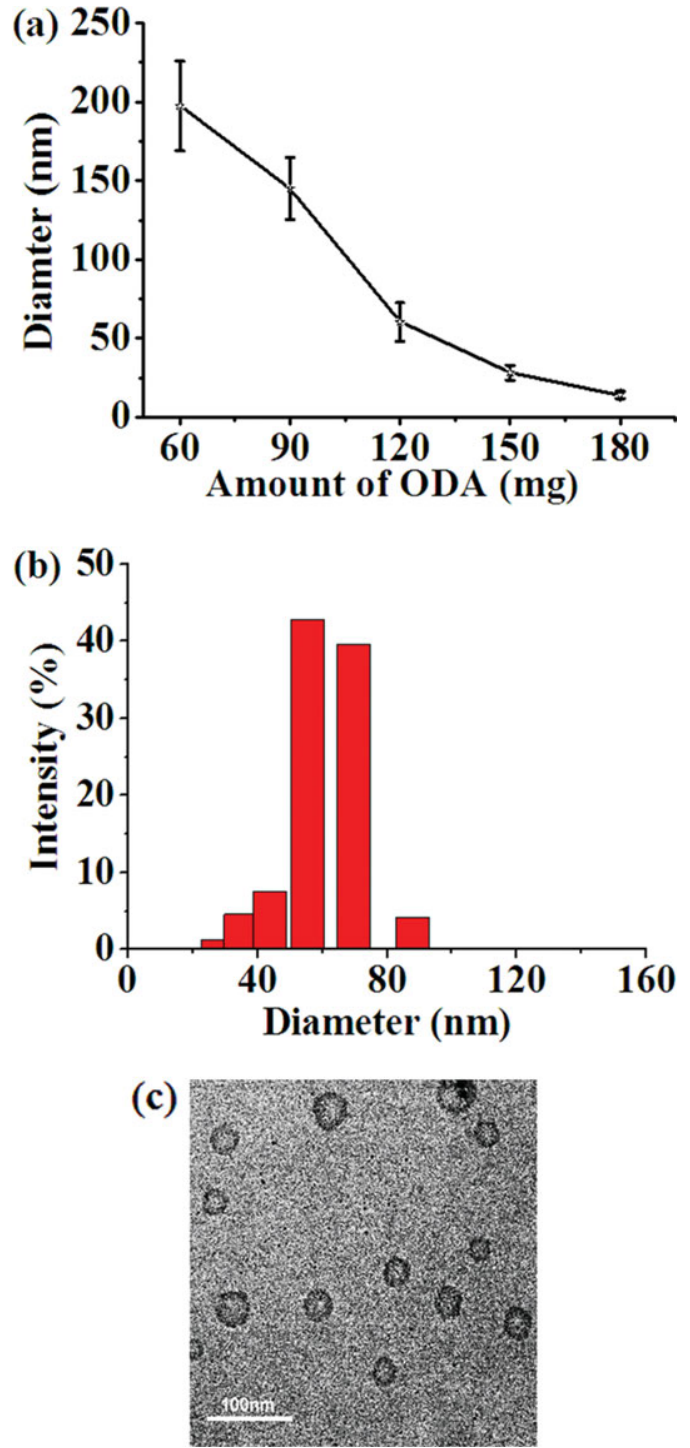

FIGURE 4. (a) Diameters of P(NIPA-co-AAm-co-ODA-co-FPA) micelles versus the amount of ODA ( $n=3$ ) (Amount of ODA was calculated by utilizing the single-factor test); (b) Size distribution of P(NIPA-co-AAmco-ODA-co-FPA) (FNM04) at $25^{\circ} \mathrm{C}$; (c) Transmittance electron microscope (TEM) picture of P(NIPA-co-AAm-co-ODA-co-FPA) (FNM04). [Color figure can be viewed in the online issue, which is available at wileyonlinelibrary.com.]

solubility in physiological saline. Insoluble fluorescein could be entrapped into the core of micelles by hydrophobic association. Since the amount of ODA residues would determine the composition of the hydrophobic core, it should control the drug loading content of the micelles. As expected, Figure 6 showed that drug loading content of micelles increased with increasing the amount of ODA. Drug loading contents of FNM07 (ODA $60 \mathrm{mg}$ ), FNM08 (ODA $90 \mathrm{mg}$ ), FNM04 (ODA $120 \mathrm{mg}$ ), FNM09 (ODA $150 \mathrm{mg}$ ), and FNM10 (ODA $180 \mathrm{mg}$ ) were $1.93 \%, 3.73 \%, 7.21 \%, 8.40 \%$, and $10.48 \%$, respectively. When there was no ODA in the feed composition, the resultant product (FNM06) displayed almost zero loading capability. In fact, this product might not be termed as micelles, because the strength of the hydrophobic interaction produced by isopropyl groups was not sufficient to stabilize the core-shell structure.

It should also be noted that after drug loading, diameter of FNM04 increased from $60 \mathrm{~nm}$ to $71.6 \mathrm{~nm}$ while its LCST decreased from $42^{\circ} \mathrm{C}$ to $41^{\circ} \mathrm{C}$.

\section{Noncovalent labeling of FNM04 with NIRD-10}

NIRD-10 was entrapped into FNM04 by hydrophobic interaction. FNM04 displayed a loading content of $1.86 \%$ for
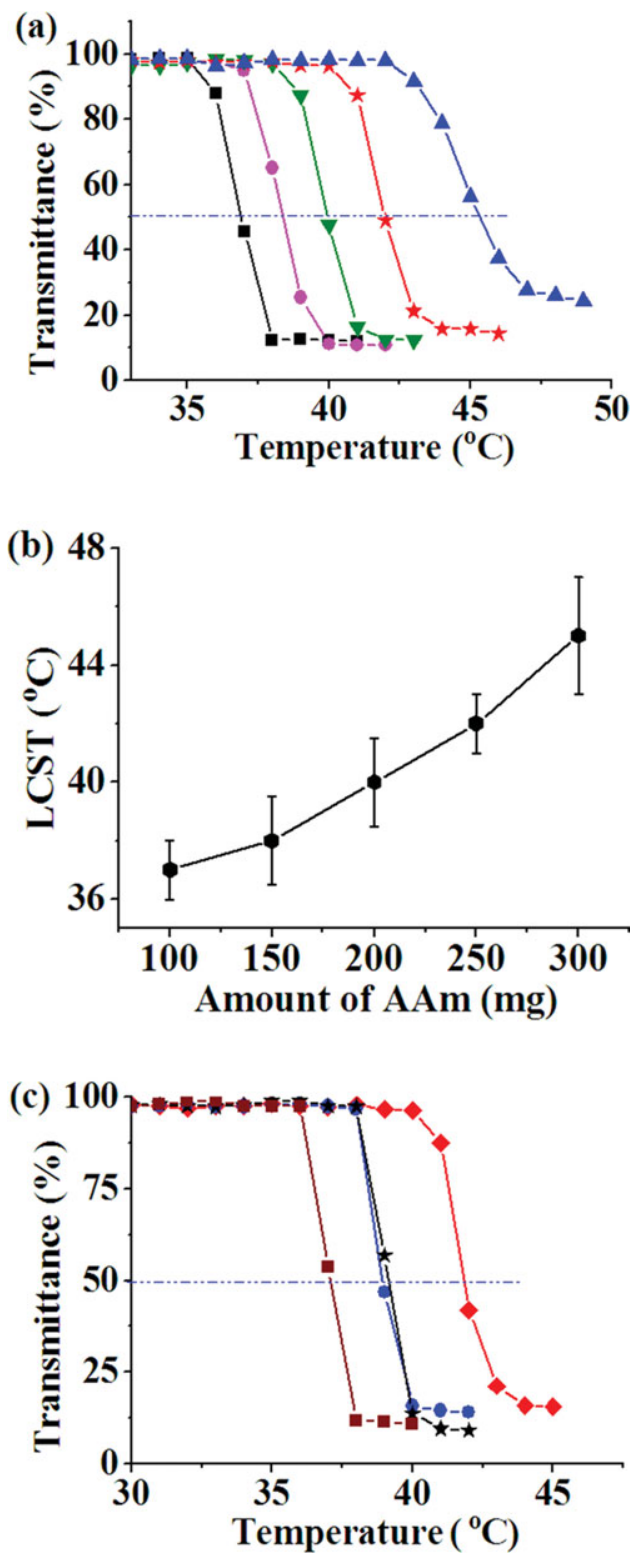

FIGURE 5. (a) Transmittance (\%) of P(NIPA-co-AAm-co-ODA-co-FPA) at different amount of AAm [100 mg (ם), $150 \mathrm{mg}(\mathbf{0}), 200 \mathrm{mg}(\boldsymbol{\nabla}), 250 \mathrm{mg}$ $(\xi)$, and $300 \mathrm{mg}(\mathbf{\Delta})$ ] versus temperature $(n=3)$ (amount of AAm was calculated by utilizing the single-factor test); (b) LCST of P(NIPAco-AAm-co-ODA-co-FPA) versus the amount of AAm $(n=3)$; (c) Transmittance (\%) of FNM04 in solutions at different ionic strength [double distilled water $(\diamond)$, Hanks simulated body fluid $(\bullet), 0.9 \% \mathrm{NaCl}(\delta)$ and $1.8 \% \mathrm{NaCl}(\square)$ ] versus temperature. [Color figure can be viewed in the online issue, which is available at wileyonlinelibrary.com.] 


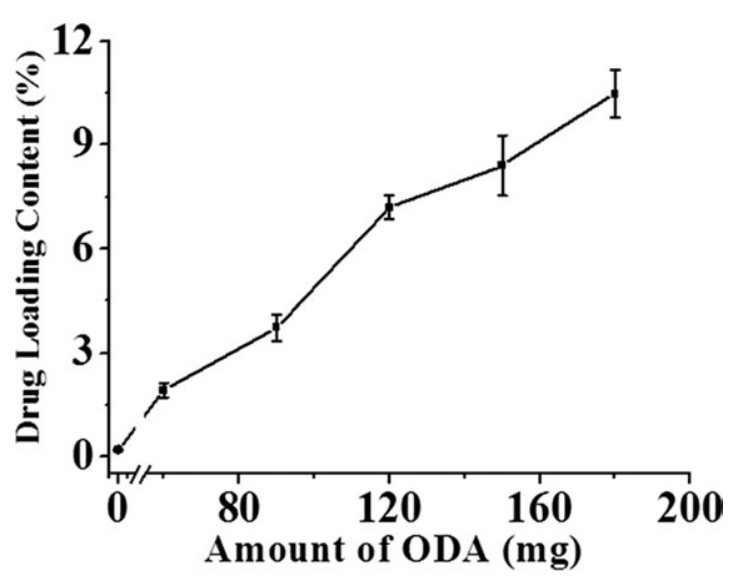

FIGURE 6. Drug (i.e., fluorescein) loading content of P(NIPA-co-AAmco-ODA-co-FPA) micelles for fluorescein versus the amount of ODA. $(n=3)$.

NIRD-10. After loading, diameter of FNM04 was increased from 60 to $65 \mathrm{~nm}$, while its LCST in double distilled water decreased from $42^{\circ} \mathrm{C}$ to $41.5^{\circ} \mathrm{C}$. Due to the accuracy of the instrument $\left( \pm 0.5^{\circ} \mathrm{C}\right)$, NIRD-10 loaded FNM04 showed the same LCST as that of FNM04 in Hanks simulated body fluid $\left(39^{\circ} \mathrm{C}\right)$.

After entrapment, red shifts of both absorbance and emission spectra of NIRD-10 were observed [Fig. 7(a,b)]. This phenomenon might be explained in terms of the stabilizing effect of the hydrophobic core of micelles for entrapped dye molecules, which could decrease the transition energy of the chromophores of NIRD-10, including a red shift of the absorption peak. The red shift of absorption peak could accordingly result in the red shift of the emission peak. The photostability of NIRD-10 was also significantly enhanced following entrapment in micelles [Fig. 7(c)].

\section{Cytotoxicity of NIRD-10 loaded FNM04}

Cytotoxicity of NIRD-10 loaded FNM04 was assessed on the ECV304 cells using the standard MTT assay. As shown in Figure 8, the dye-loaded FNM04 micelle exhibited no apparent cytotoxicity. Cell viability still remained above 95\%, even at the maximum concentration of dye-loaded FNM04 $(2.0 \mathrm{mg} / \mathrm{mL})$. The lack of cytotoxicity of NIPA-based nanomaterials was also reported by many other investigators. $^{16,17}$ Indeed, incorporation of NIPA into NIPA-based polymers could even reduce the cytotoxicity, owing to a reduction of charge density on the polymer chains. ${ }^{17}$

\section{In vivo animal evaluation}

In vivo imaging. Figure 9 illustrated showed the in vivo dynamic process of NIRD-10 loaded FNM04 in athymic nude mice assessed by the near infrared imaging system. Images of Group A [control; Fig. 9(a)] showed that free NIRD-10 yielded no targeting effect toward Bel-7402 tumor. No apparent fluorescence was observed in the tumor site at $96 \mathrm{~h}$ post-injection. Most of NIRD-10 molecules were non- specifically uptaken by liver because of their hydrophobicity. Images of Group B [the other control; Fig. 9(b)] indicated that FNM11, lack of folate ligand, displayed slightly passive retention in tumor tissue, due to the ERP effect of tumor vasculatures. However, this passive targeting was not sufficient to allow tumor diagnosis or therapy. Signal intensities in tumor and normal tissue showed no high contrast ratio throughout the entire experiment. In contrast, the NIRD-10 loaded FNM04 in group C [the experimental; Fig. 9(c)] accumulated rapidly in Bel-7402 tumor, as the fluorescence
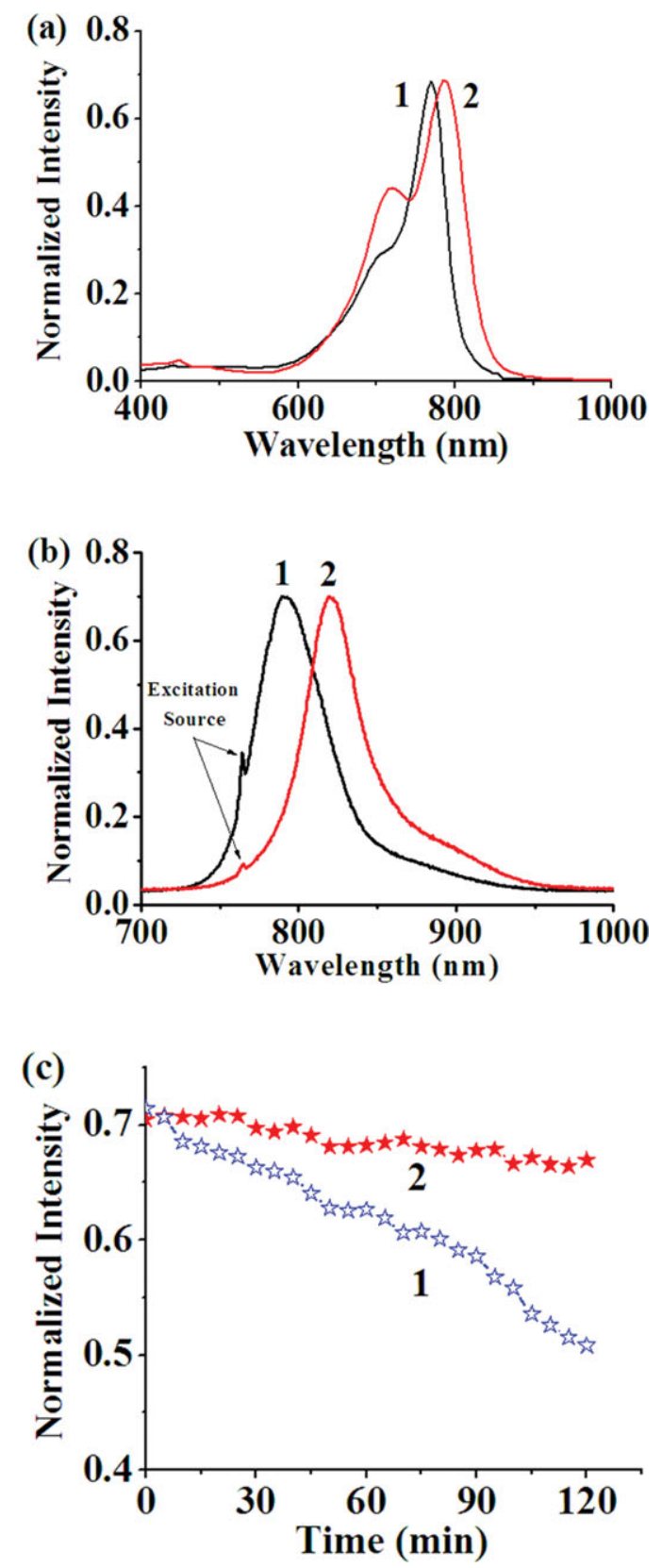

FIGURE 7. (a) Absorbance spectra of (1) free and (2) entrapped NIRD10; (b) Emission spectra of (1) free and (2) entrapped NIRD-10; (c) Photostability of (1) free and (2) entrapped NIRD-10. [Color figure can be viewed in the online issue, which is available at wileyonlinelibrary.com.] 


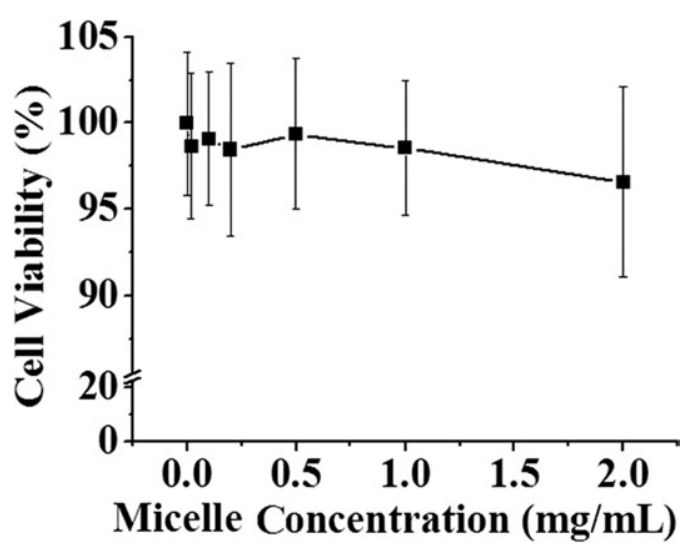

FIGURE 8. Cytotoxicity of P(NIPA-co-AAm-co-ODA-co-FPA) micelles measured by the MTT assay. $(n=6)$.

intensity in tumor persistently enhanced and reached the maximum at $2 \mathrm{~h}$ post-injection. Nevertheless, the profile of tumor was not very clear due to the systemic distribution of FNM04. As time passed by, the contrast ratio of signal intensity in tumor and normal tissue gradually augmented, and the boundary of tumor and normal tissue became relatively clear at $24 \mathrm{~h}$ post-injection. Strong fluorescence was also observed even at $96 \mathrm{~h}$ post-injection.

\section{Metabolism of FNM}

Apparent fluorescence was observed in feces collected at $3 \mathrm{~h}$ post-injection (data not shown), and this fluorescence intensity reached a peak at $12 \mathrm{~h}$ post-injection (Supporting Information Fig. 4). Furthermore, no apparent fluorescence was discovered in the urine samples throughout the entire experiment. These findings implicated that micelles were metabolized through intestine, not via kidney or urinary bladder.

\section{Ex vivo organ imaging}

Group C mice were sacrificed at $96 \mathrm{~h}$ post-injection and their main organs (heart, liver, spleen, lung, kidney, muscle, and tumor) were collected and imaged to analyze the remaining micelle particles. Figure 10 showed that there were almost no fluorescence signals in any normal organ or tissue (heart, liver, spleen, lung, kidney or muscle), and bright fluorescence was only observed in the Bel-7402 tumor tissue. The results further confirmed that FNM04 micelles possessed satisfactory targeting capability towards Bel-7402 tumor and accumulated in the tumor area for longer than $96 \mathrm{~h}$.

\section{DISCUSSION}

Conjugation of FPA

Folate could not be directly copolymerized into micelles because it possessed no " $-\mathrm{C}=\mathrm{C}-$ " chemical bond. However,

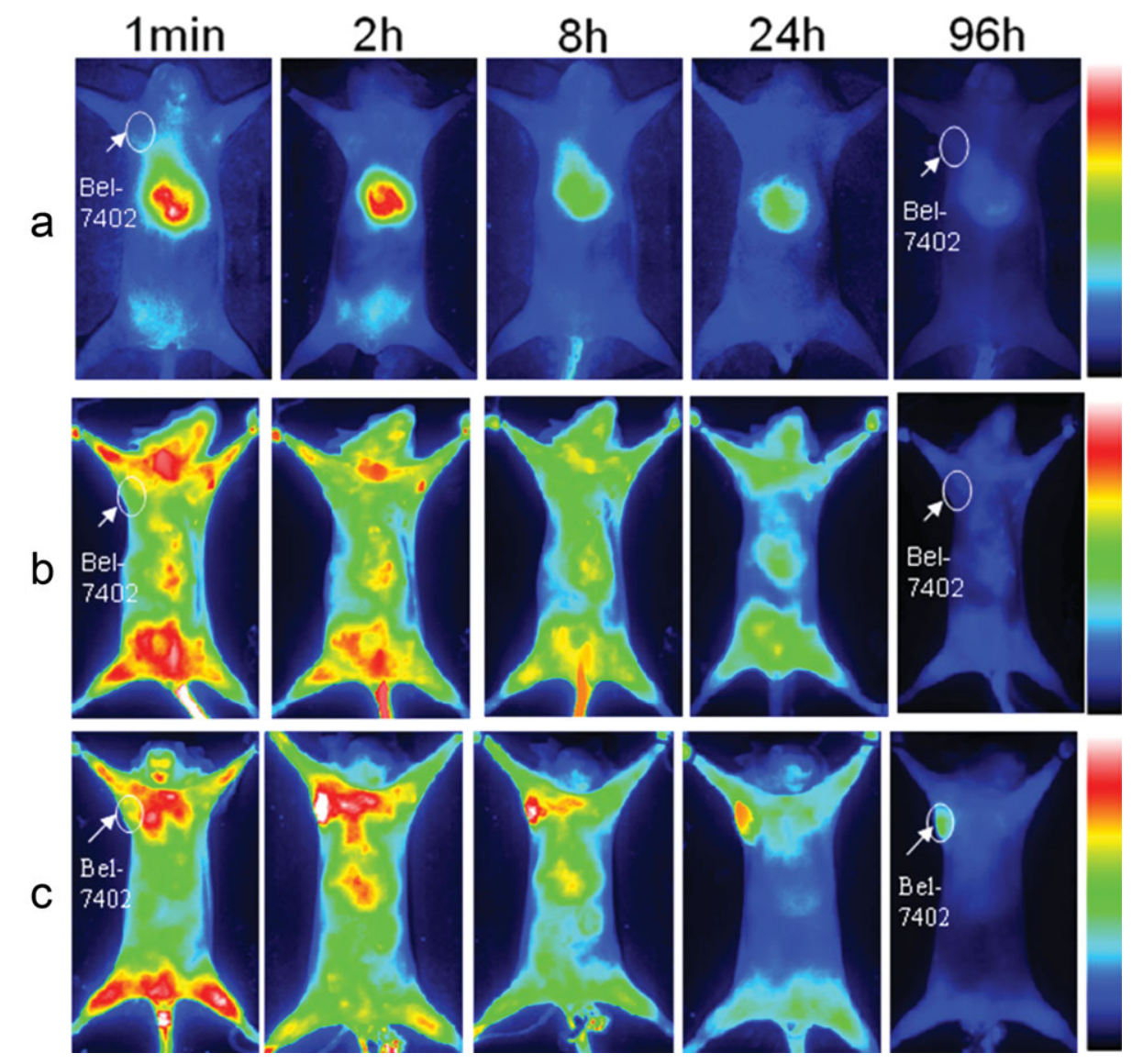

FIGURE 9. In vivo targeting behaviors of P(NIPA-co-AAm-co-ODA-co-FPA) micelles in athymic mice bearing Bel-7402 tumors: (a) free NIRD-10; (b) NIRD-10 loaded FNM11; (c) NIRD-10 loaded FNM04. ( $n=5$ per group). [Color figure can be viewed in the online issue, which is available at wileyonlinelibrary.com.] 


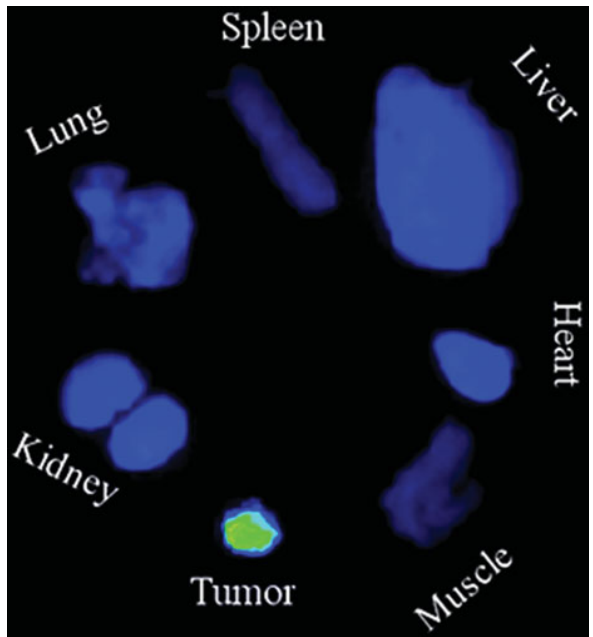

FIGURE 10. Images of organs from sacrificed Group C mice at $96 \mathrm{~h}$ post-injection. [Color figure can be viewed in the online issue, which is available at wileyonlinelibrary.com.]

with the help of amino- $\mathrm{PEG}_{200}$ as a crosslinker, folate and acrylic acid could be conjugated to form FPA. With the presence of the " $-\mathrm{C}=\mathrm{C}-$ " bond in acrylic acid (Fig. 1), the FPA could be directly copolymerized into the micelle backbone. The appearance of the characteristic peaks of folate in the absorption spectrum of FNM (Fig. 3) testified that FPA was indeed correctly synthesized. Based on these results and due to simplicity of DCC/NHS chemistry, FPA was not further characterized with Fourier transform infrared, mass spectrometry, or ${ }^{1} \mathrm{H}$ nuclear magnetic resonance.

\section{Characterization of FNM04}

Diameter is a key parameter of nanoparticles. The core-shell structure of FNM was maintained by the hydrophobic association of the ODA residues. Hence the stability of micelles was strengthened in according to the increase of ODA content, which could also produce micelles with smaller sizes. To this regard, diameter/core-shell structure of FNM was regulated by controlling the feed amount of ODA [Fig. 4(a)].

LCST is another key parameter for the thermo-responsive nanoparticles. Following systemic administration, FNM should be maintained in the swelling state at normal physiological conditions but changed to a shrinking state under hyperthermia treatment. The hydrophilicity of AAm, without the isopropyl group, is superior to that of NIPA. Hence the more AAm in micelles, the better hydrophilicity of the micelle shell and, which consequently requires more energy to destroy the core-shell structure, resulting in an increase of LCST of micelles [Fig. 5(a)]. Therefore, LCST of micelles could be regulated by altering the feed amount of AAm. Aside from the amount of AAm, ionic strength of the solution is also a crucial factor affecting the LCST of micelles. Generally, the addition of electrolytes provokes flocculation of the aqueous colloidal dispersions owing to the salting out effect. This phenomenon was also observed in our thermoresponsive micelles [Fig. 5(c)].

Based on these findings, FNM04 that possessed the most suitable diameter (about $60 \mathrm{~nm}$, in order to lower the possibility of being scavenged by the reticuloendothelial system) and LCST $\left(42^{\circ} \mathrm{C}\right.$ in double distilled water and $39^{\circ} \mathrm{C}$ in Hanks simulated body fluid) was selected for further in vitro and in vivo investigation.

\section{Noncovalent labeling of FNM04}

After entrapment by micelles, the photostability of NIRD10 was evidently enhanced [Fig. 7(c)]. This enhancement in photostability rendered NIRD-10 more suitable to trace the micelles in vivo in the mouse model with the help of near infrared technique. Administration of NIRD10 loaded micelles could effectively avoid the false identification on tumor diagnosis based on two accounts: (1) Free NIRD-10 could be rapidly cleared out from bloodstream because of its poor water-solubility; (2) NIRD-10 loaded micelles ensures the property of low leakage due to the strong binding force between NIRD-10 and ODA residues.

\section{In vivo behaviors of FNM04}

Compared with free NIRD-10, either FNM04 or FNM11, with or without folate ligand, displayed much improved systemic distribution due to the relatively better hydrophilicity of the micelle shell (Fig. 9). And the appropriate LCST $\left(39^{\circ} \mathrm{C}\right.$ in Hanks simulated body fluid) and hydrophilicy of NIRD-10 loaded FNM11 made it a competent control for the folatemediated targeting efficiency of FNM04. Intense fluorescence was observed in normal tissue for Group B or C even at $8 \mathrm{~h}$ post-injection. As a result, the nonspecific uptake of liver for micelle particles was greatly reduced, which enabled micelle particles the better opportunity to accumulate at the targeted tumor site. The results of in vivo imaging indicated that FNM04 micelles possessed the potential for tumor diagnosis and therapy.

As to the metabolism behaviors, FNM micelles could not be directly filtered by acinus renis due to their relatively large size. In addition, they were not likely degraded in vivo because of the lack of degradable bonds in the polymer backbone. Accordingly, fluorescence signals were only observed in feces, but not in urine. The micelle particles were eventually cleared by the intestine tract.

In consideration of the temperature of hyperthemia treatment (not higher than $43^{\circ} \mathrm{C}$ ) and the low grade fever of cancer patients, micelles with LCST of $40-41^{\circ} \mathrm{C}$ could be better for clinical application. And some other factors have to be taken into account, such as the temperature gradient from skin to tumor tissue and the temperature span for the complete phase transition of micelle particles. In this study, micelles with LCST of $39^{\circ} \mathrm{C}$ worked well, based on: (1) the results showed that micelles had encouraging long-circulation and targeting performance for Bel-7402 tumors; (2) the body temperature of the mouse was much lower than $39^{\circ} \mathrm{C}$ when treated with general anesthesia. And, most important, LCST of micelles is totally adjustable. Micelles with LCST of $40-41^{\circ} \mathrm{C}$ could be easily synthesized when necessary. 


\section{CONCLUSIONS}

Folate receptor targeting micelles FNM04 with diameter of about $60 \mathrm{~nm}$ and LCST of $39^{\circ} \mathrm{C}$ (in physiological condition) were successfully synthesized and characterized. The photostability of NIRD-10 was enhanced after being loaded into micelles, rendering it more suitable for in vivo real-time imaging. Active targeting by NIRD-10 loaded FNM04 was confirmed in mice bearing folate receptor expressing Bel7402 tumors, as demonstrated by the in vivo tumor imaging as well as ex vivo organ imaging. FNM04 also displayed a long circulating capability, apparently due to its hydrophilic shell. The results of dual capability of targeting and long circulation made FNM04 micelles a potential candidate for clinical application on both the diagnosis and therapy of folate receptor-positive tumors.

\section{REFERENCES}

1. Qiao P, Niu QS, Wang ZB, Cao DP. Synthesis of thermosensitive micelles based on poly ( $\mathrm{N}$-isopropylacrylamide) and poly (L-alanine) for controlled release of adriamycin. Chem Eng J 2010;159:257-263.

2. Wei $H$, Cheng $S X$, Zhang $X Z$, Zhuo $R X$. Thermo-sensitive polymeric micelles based on poly ( $N$-isopropylacrylamide) as drug carriers. Prog Polym Sci 2008;34:893-910.

3. Maeda H, Wu J, Sawa T, Matsumura Y, Hori K. Tumor vascular permeability and the EPR effect in macromolecular therapeutics: A review. J Control Release 2000;65:271-284.

4. Nasongkla N, Bey E, Ren J, Ai H, Khemtong C, Gao JS. Multifunctional polymeric micelles as cancer-targeted, MRI-ultrasensitive drug delivery systems. Nano Lett 2006;6:2427-2430.

5. Tsai HC, Chang WH, Lo CL, Tsai CH, Chang $\mathrm{CH}$, Ou TW, Yen TC, Hsiue GH. Graft and diblock copolymer multifunctional micelles for cancer chemotherapy and imaging. Biomaterials 2010;31: 2293-2301.
6. Kim D, Lee ES, Oh KT, Gao ZG, Bae YH. Doxorubicin-loaded polymeric micelle overcomes multidrug resistance of cancer by double-targeting folate receptor and early endosomal pH. Small 2008; 4:2043-2050.

7. Fares MM, Al-Shboul AM. 2012. Stimuli pH-responsive ( $N$-vinyl imidazole-co-acryloylmorpholine) hydrogels; mesoporous and nanoporous scaffolds. J Biomed Mater Res Part A 2012;100A:863-871.

8. Zhang J, Chen HY, Xu L, Gu YQ. The targeted behavior of thermally responsive nanohydrogel evaluated by NIR system in mouse model. J Control Release 2008;131:34-40.

9. Zhang J, Deng DW, Qian ZY, Liu F, Chen XY, An LX. The targeting behavior of folate-nanohydrogel evaluated by near infrared imaging system in tumor-bearing mouse model. Pharam Res 2010;27:46-55.

10. Husseini GA, Pitt WG. Micelles and nanoparticles for ultrasonic drug and gene delivery. Adv Drug Deliv Rev 2008;60:1137-1152.

11. Bikram M, Gobin AM, Whitmire RE, West JL. Temperature-sensitive hydrogels with $\mathrm{SiO} 2-\mathrm{Au}$ nanoshells for controlled drug delivery. J Control Release 2007;123:219-227.

12. Wei $H$, Zhang $X Z$, Cheng $C$, Cheng $S X$, Zhuo RX. Self-assembled, thermosensitive micelles of a star block copolymer based on PMMA and PNIPAAm for controlled drug delivery. Biomaterials 2008;28:99-107.

13. Zhang J, Qian ZY, Gu YQ. In vivo anti-tumor efficacy of docetaxel-loaded thermally responsive nanohydrogel. Nanotechnology 2009;20:325102(8 pp).

14. Ntziachristos V, Bremer C, Weissleder R. Fluorescence imaging with near-infrared light: New technological advances that enable in vivo molecular imaging. Eur Radiol 2002;13:195-208.

15. Britton C. Near-infrared images using continuous, phase-modulated, and pulsed light with quantitation of blood and blood oxygenation. Ann N Y Acad Sci 1998;838:29-45.

16. Mao ZM, Ma L, Yan JY, Yan M, Gao C, Shen JC. The gene transfection efficiency of thermoresponsive $N, N, N$-trimethylchitosan chloride- $g$-poly $(N$ - isopropylacrylamide) copolymer. Biomaterials 2007;28:4488-4500.

17. Türk M, Dinçer S, Yuluğ IG, Pişkin E. In vitro transfection of HeLa cells with temperature sensitive copolymers. J Control Release 2004;96:325-340. 